\title{
Statistical Modelling and Analysis of Borehole Computer Experiment
}

\author{
Kazeem Adewale Osuolale ${ }^{1}$, Waheed Babatunde Yahya ${ }^{2}$ \\ and Babatunde Lateef Adeleke ${ }^{3}$ \\ Department of Statistics, University of Ilorin, P.M.B. 1515, Ilorin, Nigeria \\ whereisqosimadewale@gmail.com
}

\begin{abstract}
Computer experiments are popular techniques adopted in modern businesses, engineering, scientific and technological applications in the recent years. Its flexibility and wide applicability has made it more accepted than the classical physical experiments. The Design and Analysis of Computer Experiments (DACE) is fast growing in statistical experimental designs. In this work, an Orthogonal Array-based Latin Hypercube Design, that is, $O A(N, k) L H D$ was applied for the development of borehole computer experiment. A computer experiment was conducted based on the OA $(49,8)$ LHD using a borehole computer model. The borehole computer model was used to simulate the real life borehole experiment. The Gaussian stochastic process (Gasp) model was employed to mimic the computer model in order to save time that may be required by a complex computer code and for the purpose of predictions of the flow rate of water at untried inputs. The Maximum Likelihood Estimation technique was used to estimate the parameters of the Gasp model. The results obtained using the Gasp model indicated that the radius $\left(\mathrm{r}_{w}\right)$ and the hydraulic conductivity $\left(\mathrm{K}_{w}\right)$ of the borehole were the most important factors that influenced the flow rate of water from an upper aquifer to a lower one. The fitted Gasp model was found to be very efficient since it yielded exact results on the test data cases. The model development and analysis were performed in MATLAB package.
\end{abstract}

Keywords: Computer experiment, Gasp model, Orthogonal array-based Latin hypercube design, Borehole computer model, Space-filling design

\section{Introduction}

An experimental design is the selection of inputs by which to compute the output of computer experiments in order to achieve specific aims. It has a matrix of input variable $(\boldsymbol{X})$, where each column of $\boldsymbol{X}$ depicts a variable and each row is the combination of input variable values for a single experimental run. Conventional experimental designs originate from the theory of Design of Experiments (DOE) when physical experiments are performed [8] while space-filling designs are associated with computer experiments. Computer experiments are distinct from physical experiments because they have no random error and they deal with functions that are considered to have more complex behaviour. Properly designed experiments are essential for effective computer utilization. Deterministic computer experiments are more commonly employed in engineering, science and technology because the conventional physical experiments could require more time, money and some other resources to conduct. In some instances, the physical experiments could be difficult to perform. A computer experiment is an experiment performed using data obtained from a computer model instead of the physical process.

Received (June 10, 2017), Review Result (September 26, 2017), Accepted (October 8, 2017) 
The rapid growth in computer power has now made it possible to conduct deterministic experiments on simulators. The first computer experiment was reported to have been conducted by Enrico Fermi and colleagues [14] in Los Alamos in 1953 and since then, scientists in different disciplines have turned to computer experiments as a useful tool to understand their respective processes.

In this present work, a borehole computer experiment that utilizes a model which is a simple example of flow rate of water through a borehole from an upper aquifer to a lower aquifer that is separated by an impermeable rock layer was developed. The orthogonal array-based Latin hypercube design (OALHD) originally constructed by [12] is used to develop a borehole computer experiment and the Gasp model is employed to emulate a borehole computer model.

\section{Material and Methods}

The model development and analysis in this work were performed using MATLAB package. Orthogonal array Latin hypercube design (OALHD) was used to develop a borehole computer experiment through a model of flow rate of water and the Gasp model was subsequently used to emulate a borehole computer model. The results of OA $(49,8)$ LHD and its plot for bivariate projections among the eight input variables are provided in Table 1 and Figure 1 as constructed by the author in [13]. The approach employed by the author in [13] is different from the one employed in the construction of OALHD for three input variables computer experiment [10]. The borehole model has been investigated by several researchers including [16], [11], [7], [5] and [1] among others.

Table 1. OA $(49,8)$ LHD Constructed for Borehole Computer Experiment

\begin{tabular}{|l|l|l|l|l|l|l|l|}
\hline \multicolumn{7}{|c|}{ DA (49, 8) LHD } \\
\hline \multicolumn{9}{|c|}{ Design Points } \\
\hline 0.0102 & 0.0102 & 0.0102 & 0.0102 & 0.0102 & 0.0102 & 0.0102 & 0.0102 \\
\hline 0.0306 & 0.1531 & 0.1531 & 0.2959 & 0.4388 & 0.7245 & 0.1531 & 0.8673 \\
\hline 0.0510 & 0.2959 & 0.2959 & 0.5816 & 0.8673 & 0.4388 & 0.2959 & 0.7245 \\
\hline 0.0714 & 0.4388 & 0.4388 & 0.8673 & 0.2959 & 0.1531 & 0.4388 & 0.5816 \\
\hline 0.0918 & 0.5816 & 0.5816 & 0.1531 & 0.7245 & 0.8673 & 0.5816 & 0.4388 \\
\hline 0.1122 & 0.7245 & 0.7245 & 0.4388 & 0.1531 & 0.5816 & 0.7245 & 0.2959 \\
\hline 0.1327 & 0.8673 & 0.8673 & 0.7245 & 0.5816 & 0.2959 & 0.8673 & 0.1531 \\
\hline 0.1531 & 0.0306 & 0.1735 & 0.1735 & 0.3163 & 0.4592 & 0.7449 & 0.1735 \\
\hline 0.1735 & 0.1735 & 0.3163 & 0.4592 & 0.7449 & 0.1735 & 0.8878 & 0.0306 \\
\hline 0.1939 & 0.3163 & 0.4592 & 0.7449 & 0.1735 & 0.8878 & 0.0306 & 0.8878 \\
\hline 0.2143 & 0.4592 & 0.6020 & 0.0306 & 0.6020 & 0.6020 & 0.1735 & 0.7449 \\
\hline 0.2347 & 0.6020 & 0.7449 & 0.3163 & 0.0306 & 0.3163 & 0.3163 & 0.6020 \\
\hline 0.2551 & 0.7449 & 0.8878 & 0.6020 & 0.4592 & 0.0306 & 0.4592 & 0.4592 \\
\hline 0.2755 & 0.8878 & 0.0306 & 0.8878 & 0.8878 & 0.7449 & 0.6020 & 0.3163 \\
\hline 0.2959 & 0.0510 & 0.3367 & 0.3367 & 0.6224 & 0.9082 & 0.4796 & 0.3367 \\
\hline 0.3163 & 0.1939 & 0.4796 & 0.6224 & 0.0510 & 0.6224 & 0.6224 & 0.1939 \\
\hline 0.3367 & 0.3367 & 0.6224 & 0.9082 & 0.4796 & 0.3367 & 0.7653 & 0.0510 \\
\hline 0.3571 & 0.4796 & 0.7653 & 0.1939 & 0.9082 & 0.0510 & 0.9082 & 0.9082 \\
\hline 0.3776 & 0.6224 & 0.9082 & 0.4796 & 0.3367 & 0.7653 & 0.0510 & 0.7653 \\
\hline 0.3980 & 0.7653 & 0.0510 & 0.7653 & 0.7653 & 0.4796 & 0.1939 & 0.6224 \\
\hline 0.4184 & 0.9082 & 0.1939 & 0.0510 & 0.1939 & 0.1939 & 0.3367 & 0.4796 \\
\hline 0.4388 & 0.0714 & 0.5000 & 0.5000 & 0.9286 & 0.3571 & 0.2143 & 0.5000 \\
\hline 0.4592 & 0.2143 & 0.6429 & 0.7857 & 0.3571 & 0.0714 & 0.3571 & 0.3571 \\
\hline 0.4796 & 0.3571 & 0.7857 & 0.0714 & 0.7857 & 0.7857 & 0.5000 & 0.2143 \\
\hline 0.5000 & 0.5000 & 0.9286 & 0.3571 & 0.2143 & 0.5000 & 0.6429 & 0.0714 \\
\hline & & & & & & & \\
\hline
\end{tabular}




\begin{tabular}{|l|l|l|l|l|l|l|l|}
0.5204 & 0.6429 & 0.0714 & 0.6429 & 0.6429 & 0.2143 & 0.7857 & 0.9286 \\
\hline 0.5408 & 0.7857 & 0.2143 & 0.9286 & 0.0714 & 0.9286 & 0.9286 & 0.7857 \\
\hline 0.5612 & 0.9286 & 0.3571 & 0.2143 & 0.5000 & 0.6429 & 0.0714 & 0.6429 \\
\hline 0.5816 & 0.0918 & 0.6633 & 0.6633 & 0.2347 & 0.8061 & 0.9490 & 0.6633 \\
\hline 0.6020 & 0.2347 & 0.8061 & 0.9490 & 0.6633 & 0.5204 & 0.0918 & 0.5204 \\
\hline 0.6224 & 0.3776 & 0.9490 & 0.2347 & 0.0918 & 0.2347 & 0.2347 & 0.3776 \\
\hline 0.6429 & 0.5204 & 0.0918 & 0.5204 & 0.5204 & 0.9490 & 0.3776 & 0.2347 \\
\hline 0.6633 & 0.6633 & 0.2347 & 0.8061 & 0.9490 & 0.6633 & 0.5204 & 0.0918 \\
\hline 0.6837 & 0.8061 & 0.3776 & 0.0918 & 0.3776 & 0.3776 & 0.6633 & 0.9490 \\
\hline 0.7041 & 0.9490 & 0.5204 & 0.3776 & 0.8061 & 0.0918 & 0.8061 & 0.8061 \\
\hline 0.7245 & 0.1122 & 0.8265 & 0.8265 & 0.5408 & 0.2551 & 0.6837 & 0.8265 \\
\hline 0.7449 & 0.2551 & 0.9694 & 0.1122 & 0.9694 & 0.9694 & 0.8265 & 0.6837 \\
\hline 0.7653 & 0.3980 & 0.1122 & 0.3980 & 0.3980 & 0.6837 & 0.9694 & 0.5408 \\
\hline 0.7857 & 0.5408 & 0.2551 & 0.6837 & 0.8265 & 0.3980 & 0.1122 & 0.3980 \\
\hline 0.8061 & 0.6837 & 0.3980 & 0.9694 & 0.2551 & 0.1122 & 0.2551 & 0.2551 \\
\hline 0.8265 & 0.8265 & 0.5408 & 0.2551 & 0.6837 & 0.8265 & 0.3980 & 0.1122 \\
\hline 0.8469 & 0.9694 & 0.6837 & 0.5408 & 0.1122 & 0.5408 & 0.5408 & 0.9694 \\
\hline 0.8673 & 0.1327 & 0.9898 & 0.9898 & 0.8469 & 0.7041 & 0.4184 & 0.9898 \\
\hline 0.8878 & 0.2755 & 0.1327 & 0.2755 & 0.2755 & 0.4184 & 0.5612 & 0.8469 \\
\hline 0.9082 & 0.4184 & 0.2755 & 0.5612 & 0.7041 & 0.1327 & 0.7041 & 0.7041 \\
\hline 0.9286 & 0.5612 & 0.4184 & 0.8469 & 0.1327 & 0.8469 & 0.8469 & 0.5612 \\
\hline 0.9490 & 0.7041 & 0.5612 & 0.1327 & 0.5612 & 0.5612 & 0.9898 & 0.4184 \\
\hline 0.9694 & 0.8469 & 0.7041 & 0.4184 & 0.9898 & 0.2755 & 0.1327 & 0.2755 \\
\hline 0.9898 & 0.9898 & 0.8469 & 0.7041 & 0.4184 & 0.9898 & 0.2755 & 0.1327 \\
\hline & & & & & & & \\
\hline
\end{tabular}

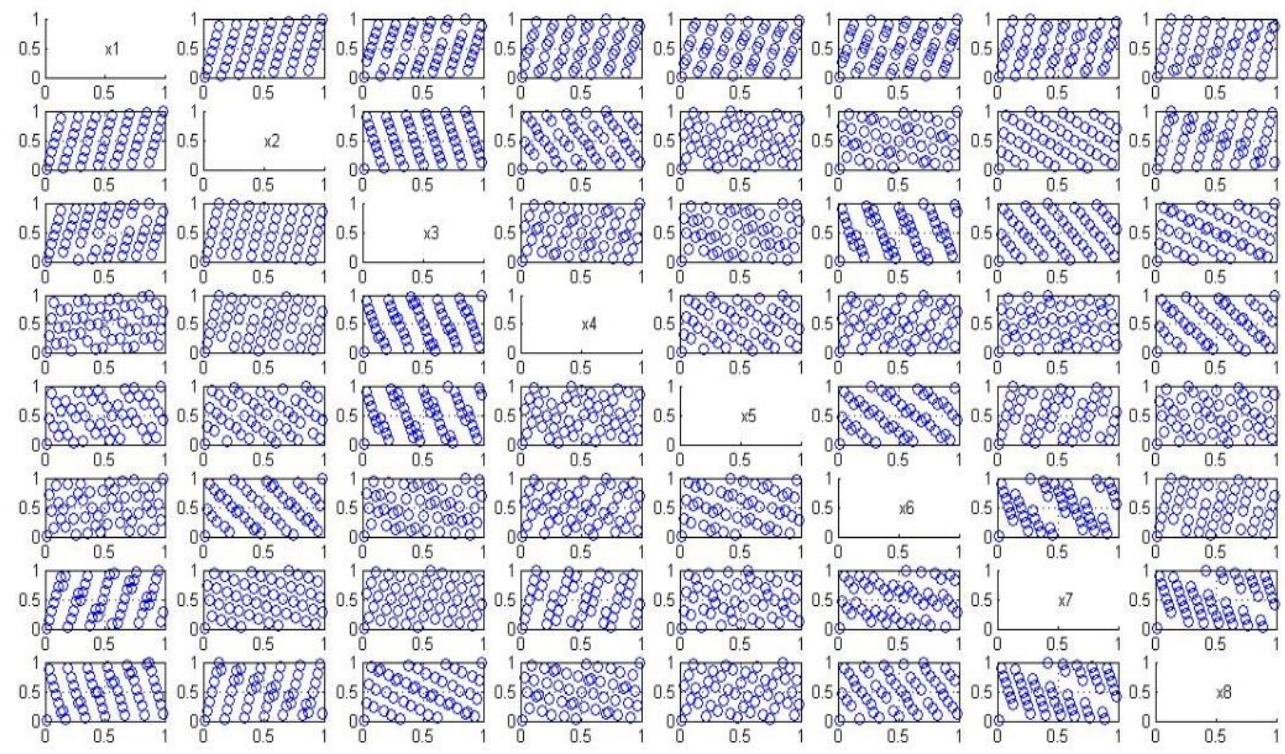

Figure 1. Projection Properties of OA $(49,8)$ LHD

The OA $(49,8)$ LHD given in Table 1 contained 49 runs and 8 input variables and was scaled according to the assumed range for design variables using Equation 1:

$$
\begin{gathered}
y_{O A L H D}=\frac{Y_{\text {data }}-Y_{\text {data }(\min )}}{Y_{\text {data }(\max )}-Y_{\text {data }(\min )}} \\
y_{\text {data }}=y_{O A L H D}\left(y_{\text {data }(\max )}-y_{\text {data }(\min )}\right)+y_{\text {data }(\min )}
\end{gathered}
$$


Table 2. Input Variables for Borehole Model

\begin{tabular}{c|lcc}
\hline Variable & Variable name & Minimum & Maximum \\
\hline $\mathrm{X}_{1}\left(r_{w}\right)$ & Radius of Borehole (metre) & 0.05 & 0.1 \\
$\mathrm{X}_{2}(r)$ & Radius of Influence (metre) & 100 & 25050 \\
$\mathrm{X}_{3}\left(T_{u}\right)$ & Transmissivity of Upper Aquifer $\left(\mathrm{m}^{2} / \mathrm{yr}\right)$ & 63070 & 89335 \\
$\mathrm{X}_{4}\left(H_{u}\right)$ & Potentiometric Head of Upper Aquifer $($ metre) & 990 & 1045 \\
$\mathrm{X}_{5}\left(T_{l}\right)$ & Transmissivity of Lower Aquifer $\left(\mathrm{m}^{2} / \mathrm{yr}\right)$ & 63.1 & 89.55 \\
$\mathrm{X}_{6}\left(H_{l}\right)$ & Potentiometric Head of Lower Aquifer(metre) & 700 & 760 \\
$\mathrm{X}_{7}(\mathrm{~L})$ & Length of Borehole (metre) & 1120 & 1400 \\
$\mathrm{X}_{8}\left(K_{w}\right)$ & Hydraulic Conductivity of Borehole (metre/yr) & 9855 & 10950 \\
$\mathrm{Y}$ & Flow Rate of Water $\left(\mathrm{m}^{3} / \mathrm{yr}\right)$ & \\
\hline
\end{tabular}

The scaled OALHD was used to develop a borehole computer experiment using the simulator in Equation 2:

$$
y=\frac{2 \pi T_{u}\left(H_{u}-H_{l}\right)}{1 n\left(r / r_{w}\right)\left[1+\frac{2 L T_{u}}{1 n\left(r / r_{w}\right) r_{w}^{2} k_{w}}+\frac{T_{u}}{T_{l}}\right]}
$$

where

$\begin{array}{lll}r_{w}(\mathrm{~m}) & = & \text { radius of borehole } \\ r(\mathrm{~m}) & = & \text { radius of influence } \\ T_{l}\left(\mathrm{~m}^{2} / \mathrm{yr}\right) & = & \text { transmissivity of lower aquifer } \\ T_{u}\left(\mathrm{~m}^{2} / \mathrm{yr}\right) & = & \text { transmissivity of upper aquifer } \\ H_{l}(\mathrm{~m}) & = & \text { potentiometric head of lower aquifer } \\ H_{u}(\mathrm{~m}) & & =\quad \text { potentiometric head of upper aquifer } \\ L(\mathrm{~m}) & & =\quad \text { length of borehole and } \\ K_{w}(\mathrm{~m} / \mathrm{yr}) & = & \text { hydraulic conductivity of borehole }\end{array}$

The scale input variables and the output from a borehole computer model constitute the experimental results for the training data sets as provided in Table 3.

Table 3. Experimental Data for Borehole Computer Experiment (Training Data Sets)

\begin{tabular}{|c|c|c|c|c|c|c|c|c|}
\hline$r_{w}$ & $R$ & $T_{u}$ & $H_{u}$ & $T_{l}$ & $H_{l}$ & $L$ & $K_{w}$ & $\mathrm{Y}$ \\
\hline 0.055 & 1397.057 & 63922.849 & 991.247 & 63.556 & 700.833 & 1123.256 & 9957.012 & 24.111 \\
\hline 0.056 & 2113.633 & 64202.654 & 991.357 & 63.561 & 700.834 & 1123.256 & 10086.946 & 25.194 \\
\hline 0.056 & 2830.210 & 64482.458 & 991.468 & 63.557 & 700.834 & 1123.256 & 10216.880 & 26.309 \\
\hline 0.057 & 3546.786 & 64762.263 & 991.320 & 63.562 & 700.833 & 1123.256 & 10346.814 & 27.433 \\
\hline 0.058 & 4263.362 & 64062.752 & 991.431 & 63.558 & 700.834 & 1123.256 & 10476.748 & 28.613 \\
\hline 0.059 & 4979.939 & 64342.556 & 991.284 & 63.563 & 700.834 & 1123.256 & 10606.682 & 29.801 \\
\hline 0.060 & 5696.515 & 64622.361 & 991.394 & 63.560 & 700.833 & 1123.256 & 10736.616 & 31.047 \\
\hline 0.061 & 2216.001 & 64082.738 & 991.289 & 63.557 & 700.833 & 1123.256 & 9975.574 & 29.671 \\
\hline 0.061 & 2932.578 & 64362.542 & 991.399 & 63.562 & 700.834 & 1123.256 & 10105.508 & 30.911 \\
\hline 0.062 & 3649.154 & 64642.347 & 991.252 & 63.559 & 700.834 & 1123.256 & 10235.442 & 32.156 \\
\hline 0.063 & 4365.731 & 63942.835 & 991.362 & 63.563 & 700.834 & 1123.256 & 10365.376 & 33.464 \\
\hline 0.064 & 5082.307 & 64222.640 & 991.473 & 63.560 & 700.833 & 1123.256 & 10495.310 & 34.806 \\
\hline 0.065 & 5798.883 & 64502.445 & 991.326 & 63.556 & 700.834 & 1123.256 & 10625.244 & 36.152 \\
\hline 0.066 & 1499.425 & 64782.249 & 991.436 & 63.561 & 700.834 & 1123.256 & 10755.178 & 37.583 \\
\hline 0.067 & 3034.946 & 64242.626 & 991.331 & 63.559 & 700.834 & 1123.256 & 9994.136 & 35.816 \\
\hline 0.067 & 3751.522 & 64522.431 & 991.441 & 63.563 & 700.833 & 1123.256 & 10124.070 & 37.219 \\
\hline 0.068 & 4468.099 & 64802.235 & 991.294 & 63.560 & 700.834 & 1123.256 & 10254.004 & 38.624 \\
\hline
\end{tabular}




\begin{tabular}{|c|c|c|c|c|c|c|c|c|}
\hline 069 & 184.675 & 64102.724 & 991.405 & 6 & 34 & & & \\
\hline 70 & 901.251 & 54382.528 & 991.257 & & & & & 41.575 \\
\hline & & & & & & & & \\
\hline & & & & & & & & \\
\hline & & & & & & & & \\
\hline & & & & & & & & \\
\hline & & & & & & & & \\
\hline 75 & & & & & & & & \\
\hline 76 & & & & & & & & \\
\hline 77 & & & & & & & & \\
\hline 0.078 & & & & & & & & \\
\hline 78 & & & & & & & & \\
\hline 79 & & & & & & & & \\
\hline 080 & & & & & & & & \\
\hline 81 & & & & & & & & \\
\hline 0.082 & & & & & & & & \\
\hline 83 & & & & & & & & \\
\hline 84 & & & & & & & & \\
\hline 84 & & & & & & & & \\
\hline & & & & & & & & \\
\hline & & & & & & & & \\
\hline & & & & & & & & \\
\hline & & & & & & & & \\
\hline 0.089 & & & & & & & & \\
\hline 0.089 & & & & & & & & \\
\hline 0.090 & & & & & & & & \\
\hline 0.091 & & & & & & & & 372 \\
\hline 0.092 & 2727.842 & 64462.472 & 991.462 & & & & 10328.252 & 70.538 \\
\hline 0.093 & 344 & 64 & 99 & 6 & & & & 687 \\
\hline 0.094 & 4160.994 & & 991. & & & & & 74.945 \\
\hline & & & & & & & & \\
\hline 0.095 & 594.147 & & 991.389 & 3550 & 700.833 & 256 & 10847.988 & \\
\hline
\end{tabular}

\section{Modelling Borehole Computer Experiment}

A borehole computer model is a model of a physical process to be emulated using a metamodel. A metamodel is referred to as a model used to emulate a borehole computer model. A wide variety of techniques have been discussed in the literature for creating the metamodels [14]. These techniques include response surface modelling [9], Radial Basis Functions ([2]; [3]), Multivariate Adaptive Regression Splines [4] and Support Vector Machine [15]. In this study, a Gaussian stochastic process (Gasp) model was investigated as an alternative technique for approximating a borehole computer model. The Gasp model allows a wide range of correlation functions $R\left(X_{v} X^{\prime}\right)$ to be used. A Gaussian correlation function was chosen in this study. The output of the borehole computer experimental data was modelled using a Gaussian stochastic process model as described below:

$$
\begin{gathered}
Y(x)=\sum_{j=1}^{k} \beta_{j} f_{j}(x)+Z(x) \\
\beta_{\mathrm{j}} \mathrm{f}_{\mathrm{j}}(\mathrm{x})=\beta_{1} \mathrm{f}_{1}(\mathrm{x})+\beta_{2} \mathrm{f}_{2}(\mathrm{x})+\cdots \beta_{\mathrm{k}} \mathrm{f}_{\mathrm{k}}(\mathrm{x}) \\
=\left[\mathrm{f}_{1}(\mathrm{x})+\mathrm{f}_{2}(\mathrm{x})+\cdots f_{\mathrm{k}}(x)\right] \beta_{\mathrm{j}}
\end{gathered}
$$




$$
=\mathrm{f}(x)^{\mathrm{T}} \beta_{\mathrm{j}}
$$

where $f_{1}(x), \ldots, f_{k}(x)$ are ${ }^{k}$ known regression functions and $\beta_{1}, \ldots, \beta_{k}$ are their corresponding (unknown) parameters and $Z(x)$ is a stochastic process which is assumed to have mean zero and variance-covariance structure

$$
\operatorname{Cov}\left(Z(x), Z\left(x^{r}\right)\right)=\sigma_{z}^{2} R\left(X, X^{\prime}\right)
$$

$\sigma_{z}^{2}$ is the process variance and $R\left(X_{r} X^{\prime}\right)$ is the Gaussian correlation function that can be tuned to the data. The Gaussian correlation function is given as

$$
R\left(X, X^{\prime}\right)=\prod_{j=1}^{d} \exp \left(-\theta_{j}\left|x_{j}-x_{j}{ }^{\prime}\right|^{2}\right)
$$

where $\theta_{j} \geq 0$. The parameter ${ }^{\theta}$ is important in the correlation structure of $\mathrm{Z}$. When ${ }^{\theta}$ is large there is a small correlation between observations and therefore prediction is more difficult whereas there is a large correlation between observations and prediction is much simpler when ${ }^{\theta}$ is small. The selection of the correlation function is very useful in the prediction process. The author in [6] discussed the effects of ${ }^{\theta}$ on the prediction of output of a computer experiment. The correlation matrix, $\mathrm{R}$ is given as an $(n \times n)$ matrix given in Equation 7:

$$
\boldsymbol{R}=\left[\begin{array}{ccc}
R\left(x_{1}, x_{1}\right) & \cdots & R\left(x_{1}, x_{n}\right) \\
\vdots & \ddots & \vdots \\
R\left(x_{n}, x_{1}\right) & \cdots & R\left(x_{n}, x_{n}\right)
\end{array}\right]
$$

The matrix $R$ is symmetric since $R\left(x_{i}, x_{j}\right)=R\left(x_{j}, x_{i}\right)$ and the diagonal consists of all ones because $R\left(x_{i}, x_{i}\right)=1$. The correlation between an unknown point $x$ and the $n$ known sample points is given by the vector:

$$
r_{x}=\left[R\left(x_{1}, x\right), \ldots, R\left(x_{n}, x\right)\right]^{T}
$$

The best Linear Unbiased Predictor (BLUP) is obtained by minimizing the mean square error of the predictions. The BLUP at an untried point $x$ is therefore given as:

$$
\hat{y}(x)=f_{x}^{T} \hat{\beta}+r^{T}(x) R^{-1}(y-F \hat{\beta})
$$

where $\mathrm{F}$ is the expanded design matrix $n \times k$ given by

$$
\begin{aligned}
& F=\left(\begin{array}{l}
f^{T}\left(x_{1}\right) \\
\ldots \ldots . . \\
f^{T}\left(x_{n}\right)
\end{array}\right) \\
& f(x)=\left[f_{1}(x), \ldots, f_{k}(x)\right]^{T}
\end{aligned}
$$

The Maximum Likelihood Estimation (MLE) method was used to estimate the Gasp model parameters $(\beta, \theta$ and $\sigma)$ and it is an objective estimator that is most consistent with the observed data. The MLE assumes the residuals have a known probability distribution shape, 
that is, the Gaussian probability distribution. The correlation parameter $\theta$ was found using mlegp package in $\mathrm{R}$ software. The MLE estimation of $\beta$ equals its least-squares estimate and is given by

$$
\hat{\beta}=\left(F^{T} R^{-1} F\right)^{-1} F^{T} R^{-1} y
$$

and the MLE of the process variance is also given by

$$
\hat{\sigma}_{z}^{2}=\frac{1}{n}(y-F \hat{\beta})^{T} R^{-1}(y-F \hat{\beta})
$$

\section{Analysis of Borehole Computer Experiment}

The training datasets given in Table 3 showed that the eight input variables involved in the borehole computer experiment were of different scales. These variables were normalized by subtracting their means and multiplying by the reciprocal of their standard deviations before the analysis. This is required to lessen the dimension effect of each design variable and avert the Gasp model from being inconsistent in prediction. The normalized experimental data based on the borehole computer experiment and the estimated results are given in Table 4 and Table 5, respectively.

\begin{tabular}{|c|c|c|c|c|c|c|c|c|}
\hline $\mathrm{x}_{1}$ & $\mathrm{X}_{2}$ & $\mathrm{X}_{3}$ & $\mathrm{X}_{4}$ & $\mathrm{X}_{5}$ & $\mathrm{X}_{6}$ & $\mathrm{X}_{7}$ & $\mathrm{X}_{8}$ & $\bar{Y}$ \\
\hline-1.680 & -1.680 & -1.680 & -1.680 & -1.680 & -1.680 & -1.680 & -1.680 & -1.565 \\
\hline-1.610 & 1.190 & -0.700 & -0.210 & 0.280 & 0.770 & 1.260 & -1.190 & -1.495 \\
\hline-1.540 & .700 & 0.280 & 1.260 & -1.190 & -0.210 & 0.770 & -0.700 & -1.424 \\
\hline-1.470 & -0.210 & 1.260 & -0.700 & 0.770 & -1.190 & 0.280 & -0.210 & -1.353 \\
\hline-1.400 & 0.280 & -1.190 & 0.770 & -0.700 & 1.260 & -0.210 & 0.280 & -1.277 \\
\hline-1.330 & 0.770 & -0.210 & -1.190 & 1.260 & 0.280 & -0.700 & 0.770 & -1.201 \\
\hline-1.260 & 1.260 & 0.770 & 0.280 & -0.210 & -0.700 & -1.190 & 1.260 & -1.122 \\
\hline-1.190 & .120 & -1.120 & -1.120 & -1.120 & -1.120 & -1.120 & -1.610 & -1.210 \\
\hline-1.120 & -0.630 & -0.140 & 0.350 & 0.840 & 1.330 & -1.610 & -1.120 & -1.131 \\
\hline-1.050 & -0.140 & 0.840 & -1.610 & -0.630 & 0.350 & 1.330 & -0.630 & -1.051 \\
\hline-0.980 & 0.350 & -1.610 & -0.140 & 1.330 & -0.630 & 0.840 & -0.140 & -0.968 \\
\hline-0.910 & 0.840 & -0.630 & 1.330 & -0.140 & -1.610 & 0.350 & 0.350 & -0.882 \\
\hline-0.840 & 1.330 & 0.350 & -0.630 & -1.610 & 0.840 & -0.140 & 0.840 & -0.796 \\
\hline-0.770 & 610 & 1.330 & 0.840 & 50 & -0.140 & -0.630 & 1.330 & -0.705 \\
\hline-0.700 & -0.560 & -0.560 & -0.560 & -0.560 & -0.560 & -0.560 & -1.540 & -0.818 \\
\hline-0.630 & 0.070 & 0.420 & 0.910 & .400 & -1.540 & -1.050 & -1.050 & -0.728 \\
\hline-0.560 & 0.420 & 1.400 & -1.050 & -0.070 & 0.910 & -1.540 & -0.560 & -0.638 \\
\hline-0.490 & 0.910 & -1.050 & 0.420 & -1.540 & -0.070 & 1.400 & -0.070 & -0.544 \\
\hline-0.420 & 1.400 & -0.070 & -1.540 & 0.420 & -1.050 & 0.910 & 0.420 & -0.450 \\
\hline-0.350 & 540 & 0.910 & -0.070 & -1.050 & 1.400 & 0.420 & 0.910 & -0.350 \\
\hline-0.280 & -1.050 & -1.540 & 1.400 & 0.910 & 0.420 & -0.070 & 1.400 & -0.249 \\
\hline-0.210 & 0.000 & 0.000 & 0.000 & 0.000 & 0.000 & 0.000 & -1.470 & -0.388 \\
\hline-0.140 & 0.490 & 0.980 & 1.470 & -1.470 & -0.980 & -0.490 & -0.980 & -0.288 \\
\hline-0.070 & 0.980 & -1.470 & -0.490 & 0.490 & 1.470 & -0.980 & -0.490 & -0.187 \\
\hline 0.000 & 1.470 & -0.490 & 0.980 & -0.980 & 0.490 & -1.470 & 0.000 & -0.082 \\
\hline 0.070 & -1.470 & 0.490 & -0.980 & 0.980 & -0.490 & 1.470 & 0.490 & 0.025 \\
\hline 0.140 & -0.980 & 1.470 & 0.490 & -0.490 & -1.470 & 0.980 & 0.980 & 0.135 \\
\hline 0.210 & -0.490 & -0.980 & -1.470 & 1.470 & 0.980 & 0.490 & 1.470 & 0.244 \\
\hline 0.280 & 0.560 & 0.560 & 0.560 & 0.560 & 0.560 & 0.560 & -1.400 & 0.079 \\
\hline 0.350 & 1.050 & 1.540 & -1.400 & -0.910 & -0.420 & 0.070 & -0.910 & 0.188 \\
\hline 0.420 & 1.540 & -0.910 & 0.070 & 1.050 & -1.400 & -0.420 & -0.420 & 0.302 \\
\hline 0.490 & -1.400 & 0.070 & 1.540 & -0.420 & 1.050 & -0.910 & 0.070 & 0.421 \\
\hline
\end{tabular}

Table 4. Normalized Experimental Data for Borehole Computer Experiment 


\begin{tabular}{|r|r|r|r|r|r|r|r|r|}
0.560 & -0.910 & 1.050 & -0.420 & 1.540 & 0.070 & -1.400 & 0.560 & 0.537 \\
\hline 0.630 & -0.420 & -1.400 & 1.050 & 0.070 & -0.910 & 1.540 & 1.050 & 0.659 \\
\hline 0.700 & 0.070 & -0.420 & -0.910 & -1.400 & 1.540 & 1.050 & 1.540 & 0.780 \\
\hline 0.770 & 1.120 & 1.120 & 1.120 & 1.120 & 1.120 & 1.120 & -1.330 & 0.583 \\
\hline 0.840 & 1.610 & -1.330 & -0.840 & -0.350 & 0.140 & 0.630 & -0.840 & 0.703 \\
\hline 0.910 & -1.330 & -0.350 & 0.630 & 1.610 & -0.840 & 0.140 & -0.350 & 0.832 \\
\hline 0.980 & -0.840 & 0.630 & -1.330 & 0.140 & 1.610 & -0.350 & 0.140 & 0.957 \\
\hline 1.050 & -0.350 & 1.610 & 0.140 & -1.330 & 0.630 & -0.840 & 0.630 & 1.089 \\
\hline 1.120 & 0.140 & -0.840 & 1.610 & 0.630 & -0.350 & -1.330 & 1.120 & 1.223 \\
\hline 1.190 & 0.630 & 0.140 & -0.350 & -0.840 & -1.330 & 1.610 & 1.610 & 1.356 \\
\hline 1.260 & 1.680 & 1.680 & 1.680 & 1.680 & 1.680 & 1.680 & -1.260 & 1.125 \\
\hline 1.330 & -1.260 & -0.770 & -0.280 & 0.210 & 0.700 & 1.190 & -0.770 & 1.260 \\
\hline 1.400 & -0.770 & 0.210 & 1.190 & -1.260 & -0.280 & 0.700 & -0.280 & 1.398 \\
\hline 1.470 & -0.280 & 1.190 & -0.770 & 0.700 & -1.260 & 0.210 & 0.210 & 1.535 \\
\hline 1.540 & 0.210 & -1.260 & 0.700 & -0.770 & 1.190 & -0.280 & 0.700 & 1.679 \\
\hline 1.610 & 0.700 & -0.280 & -1.260 & 1.190 & 0.210 & -0.770 & 1.190 & 1.822 \\
\hline 1.680 & 1.190 & 0.700 & 0.210 & -0.280 & -0.770 & -1.260 & 1.680 & 1.972 \\
\hline
\end{tabular}

Table 5. Results of the Estimated Model for Borehole Computer Experiment

\begin{tabular}{|c|c|c|}
\hline Model Parameters & Estimated Values & P-values \\
\hline$\hat{\beta}_{0}$ & 0.8150 & $<0.001$ \\
\hline$\hat{\beta}_{1}$ & 0.9425 & 0.9692 \\
\hline$\hat{\beta}_{2}$ & $-4.6449 \mathrm{e}-04$ & 0.9401 \\
\hline$\hat{\beta}_{3}$ & $-7.9773 \mathrm{e}-06$ & 0.1442 \\
\hline$\hat{\beta}_{4}$ & $8.3103 \mathrm{e}-04$ & 0.9841 \\
\hline$\hat{\beta}_{5}$ & $-1.1769 \mathrm{e}-05$ & 0.9475 \\
\hline$\hat{\beta}_{6}$ & $-1.5467 \mathrm{e}-04$ & 0.9281 \\
\hline$\hat{\beta}_{7}$ & $1.0115 \mathrm{e}-05$ & 0.0540 \\
\hline$\hat{\beta}_{8}$ & 0.0727 & \\
\hline$\hat{\sigma}_{z}^{2}$ & 0.1018 & \\
\hline
\end{tabular}

The estimated linear main effects $\hat{\beta}_{i}, i=1,2,3,4,5,6,7$ and 8 (corresponding to $r_{w}, r$, $T_{u}, H_{u}, T_{l}, H_{l}, L$ and $K_{w}$, respectively) of the borehole computer model were presented in Table 5 with their respective $p$-values for the t-test for $i=1, \ldots, 8$ and $\widehat{\partial}_{z}^{2}$. The linear main effects for $r_{w}(0.9425)$ and $K_{w}(0.0727)$ are relatively large while their respective $p$-values $<0.001$ and 0.0540 are quite small. This implies that, the radius of borehole $\left(r_{w}\right)$ is highly significant and its hydraulic conductivity $\left(K_{w}\right)$ is also significant with $p$-value $=0.0540$. The estimated values for the radius $\left(r_{w}\right)$ and the hydraulic conductivity of borehole $\left(K_{w}\right)$ have the same signs, indicating that $r_{w}$ and $K_{w}$ have the same effects on the flow rate of water.

For the sake of prediction, additional simulations were performed to verify the accuracy of the Gasp model since the goodness of fit obtained from the training datasets may not be sufficient to assess the accuracy of newly predicted points. The additional simulations constitute the test datasets. The assumed range for the test data is given in Table 6 and the normalized experimental data is also given in Table 7. 
Table 6. Input and Output Variables for Borehole Model (Test data)

\begin{tabular}{c|lll}
\hline Variable & \multicolumn{1}{|c}{ Variable Name } & \multicolumn{1}{c}{ Minimum } & \multicolumn{1}{c}{ Maximum } \\
\hline $\mathrm{X}_{1}$ & Radius of Borehole (metre) & 0.11 & 0.15 \\
$\mathrm{X}_{2}$ & Radius of Influence (metre) & 25051 & $50 \mathrm{e} 3$ \\
$\mathrm{X}_{3}$ & Transmissivity of Upper Aquifer $\left(\mathrm{m}^{2} / \mathrm{yr}.\right)$ & 89336 & 115600 \\
$\mathrm{X}_{4}$ & Potentiometric Head of Upper Aquifer (metre) & 1046 & 1100 \\
$\mathrm{X}_{5}$ & Transmissivity of Lower Aquifer (m²/yr.) & 89.56 & 116 \\
$\mathrm{X}_{6}$ & Potentiometric Head of Lower Aquifer(metre) & 761 & 820 \\
$\mathrm{X}_{7}$ & Length of Borehole (metre) & 1401 & 1680 \\
$\mathrm{X}_{8}$ & Hydraulic Conductivity of Borehole (metre/yr.) & 10951 & 12045 \\
$\mathrm{Y}$ & Flow Rate of Water (m $3 / \mathrm{yr}$.) & - & - \\
\hline
\end{tabular}

Table 7. Normalized Experimental Data for Borehole Computer Experiment

\begin{tabular}{|c|c|c|c|c|c|c|c|c|}
\hline $\mathrm{X}_{1}$ & $\mathrm{X}_{2}$ & $\mathrm{x}_{3}$ & $\mathrm{X}_{4}$ & $\mathrm{X}_{5}$ & $\mathrm{X}_{6}$ & $x_{7}$ & $\mathrm{X}_{8}$ & $\mathrm{y}$ \\
\hline-1.680 & -1.680 & -1.680 & -1.680 & -1.680 & -1.680 & -1.680 & -1.680 & -1.725 \\
\hline-1.610 & -1.190 & -0.700 & -0.210 & 0.280 & 0.770 & 1.260 & -1.190 & -1.611 \\
\hline-1.540 & -0.700 & 0.280 & 1.260 & -1.190 & -0.210 & 0.770 & -0.700 & -1.496 \\
\hline-1.470 & -0.210 & 1.260 & -0.700 & 0.770 & -1.190 & 0.280 & -0.210 & -1.383 \\
\hline-1.400 & 0.280 & -1.190 & 0.770 & -0.700 & 1.260 & -0.210 & 0.280 & -1.265 \\
\hline-1.330 & 0.770 & -0.210 & -1.190 & 1.260 & 0.280 & -0.700 & 0.770 & -1.149 \\
\hline-1.260 & 1.260 & 0.770 & 0.280 & -0.210 & -0.700 & -1.190 & 1.260 & -1.027 \\
\hline-1.190 & -1.120 & -1.120 & -1.120 & -1.120 & -1.120 & -1.120 & -1.610 & -1.316 \\
\hline-1.120 & -0.630 & -0.140 & 0.350 & 0.840 & 1.330 & -1.610 & -1.120 & -1.195 \\
\hline-1.050 & -0.140 & 0.840 & -1.610 & -0.630 & 0.350 & 1.330 & -0.630 & -1.077 \\
\hline-0.980 & 0.350 & -1.610 & -0.140 & 1.330 & -0.630 & 0.840 & -0.140 & -0.953 \\
\hline-0.910 & 0.840 & -0.630 & 1.330 & -0.140 & -1.610 & 0.350 & .350 & -0.827 \\
\hline-0.840 & 1.330 & 0.350 & -0.630 & -1.610 & 0.840 & -0.140 & 0.840 & -0.704 \\
\hline-0.770 & -1.610 & 1.330 & 0.840 & 0.350 & -0.140 & -0.630 & .330 & -0.574 \\
\hline-0.700 & -0.560 & -0.560 & -0.560 & -0.560 & -0.560 & -0.560 & -1.540 & -0.889 \\
\hline-0.630 & -0.070 & 0.420 & 0.910 & 1.400 & -1.540 & -1.050 & -1.050 & -0.761 \\
\hline-0.560 & 0.420 & 1.400 & -1.050 & -0.070 & 0.910 & -1.540 & -0.560 & -0.636 \\
\hline-0.490 & 0.910 & -1.050 & 0.420 & -1.540 & -0.070 & 1.400 & -0.070 & -0.504 \\
\hline-0.420 & 1.400 & -0.070 & -1.540 & 0.420 & -1.050 & 0.910 & 0.420 & -0.376 \\
\hline-0.350 & -1.540 & 0.910 & -0.070 & -1.050 & 1.400 & 0.420 & 0.910 & -0.240 \\
\hline-0.280 & -1.050 & -1.540 & 1.400 & 0.910 & 0.420 & -0.070 & 1.400 & -0.103 \\
\hline-0.210 & 0.000 & 0.000 & 0.000 & 0.000 & 0.000 & 0.000 & -1.470 & -0.445 \\
\hline-0.140 & 0.490 & 0.980 & 1.470 & -1.470 & -0.980 & -0.490 & -0.980 & -0.310 \\
\hline-0.070 & 0.980 & -1.470 & -0.490 & 0.490 & 1.470 & -0.980 & -0.490 & -0.177 \\
\hline 0.000 & 1.470 & -0.490 & 0.980 & -0.980 & 0.490 & -1.470 & 0.000 & -0.038 \\
\hline 0.070 & -1.470 & 0.490 & -0.980 & 0.980 & -0.490 & 1.470 & 0.490 & 0.098 \\
\hline 0.140 & -0.980 & 1.470 & 0.490 & -0.490 & -1.470 & 0.980 & 0.980 & 0.242 \\
\hline 0.210 & -0.490 & -0.980 & -1.470 & 1.470 & 0.980 & 0.490 & 1.470 & 0.381 \\
\hline 0.280 & 0.560 & 0.560 & 0.560 & 0.560 & 0.560 & 0.560 & -1.400 & 0.016 \\
\hline 0.350 & 1.050 & 1.540 & -1.400 & -0.910 & -0.420 & 0.070 & -0.910 & 0.154 \\
\hline 0.420 & 1.540 & -0.910 & 0.070 & 1.050 & -1.400 & -0.420 & -0.420 & 0.299 \\
\hline 0.490 & -1.400 & 0.070 & 1.540 & -0.420 & 1.050 & -0.910 & 0.070 & 0.447 \\
\hline 0.560 & -0.910 & 1.050 & -0.420 & 1.540 & 0.070 & -1.400 & 0.560 & 0.590 \\
\hline 0.630 & -0.420 & -1.400 & 1.050 & 0.070 & -0.910 & 1.540 & 1.050 & 0.741 \\
\hline 0.700 & 0.070 & -0.420 & -0.910 & -1.400 & 1.540 & 1.050 & 1.540 & 0.888 \\
\hline 0.770 & 1.120 & 1.120 & 1.120 & 1.120 & 1.120 & 1.120 & -1.330 & 0.495 \\
\hline 0.840 & 1.610 & -1.330 & -0.840 & -0.350 & 0.140 & 0.630 & -0.840 & 0.640 \\
\hline 0.910 & -1.330 & -0.350 & 0.630 & 1.610 & -0.840 & 0.140 & -0.350 & 0.794 \\
\hline 0.980 & -0.840 & 0.630 & -1.330 & 0.140 & 1.610 & -0.350 & 0.140 & 0.943 \\
\hline 1.050 & -0.350 & 1.610 & 0.140 & -1.330 & 0.630 & -0.840 & 0.630 & 1.100 \\
\hline 1.120 & 0.140 & -0.840 & 1.610 & 0.630 & -0.350 & -1.330 & 1.120 & 1.259 \\
\hline
\end{tabular}




\begin{tabular}{|r|r|r|r|r|r|r|r|r|}
\hline 1.190 & 0.630 & 0.140 & -0.350 & -0.840 & -1.330 & 1.610 & 1.610 & 1.414 \\
\hline 1.260 & 1.680 & 1.680 & 1.680 & 1.680 & 1.680 & 1.680 & -1.260 & 0.991 \\
\hline 1.330 & -1.260 & -0.770 & -0.280 & 0.210 & 0.700 & 1.190 & -0.770 & 1.145 \\
\hline 1.400 & -0.770 & 0.210 & 1.190 & -1.260 & -0.280 & 0.700 & -0.280 & 1.306 \\
\hline 1.470 & -0.280 & 1.190 & -0.770 & 0.700 & -1.260 & 0.210 & 0.210 & 1.463 \\
\hline 1.540 & 0.210 & -1.260 & 0.700 & -0.770 & 1.190 & -0.280 & 0.700 & 1.628 \\
\hline 1.610 & 0.700 & -0.280 & -1.260 & 1.190 & 0.210 & -0.770 & 1.190 & 1.788 \\
\hline 1.680 & 1.190 & 0.700 & 0.210 & -0.280 & -0.770 & -1.260 & 1.680 & 1.958 \\
\hline
\end{tabular}

Based on the data in Table 7, the predicted flow rate of water, y is calculated using Equation 9 and the Gasp model interpolates the test data, that is, the predicted values at untried inputs gave the same results as the simulated values. This quality makes Gasp model approximately an exact interpolator. The graph of the predicted versus simulated output of the borehole model is given in Figure 2 as shown below:

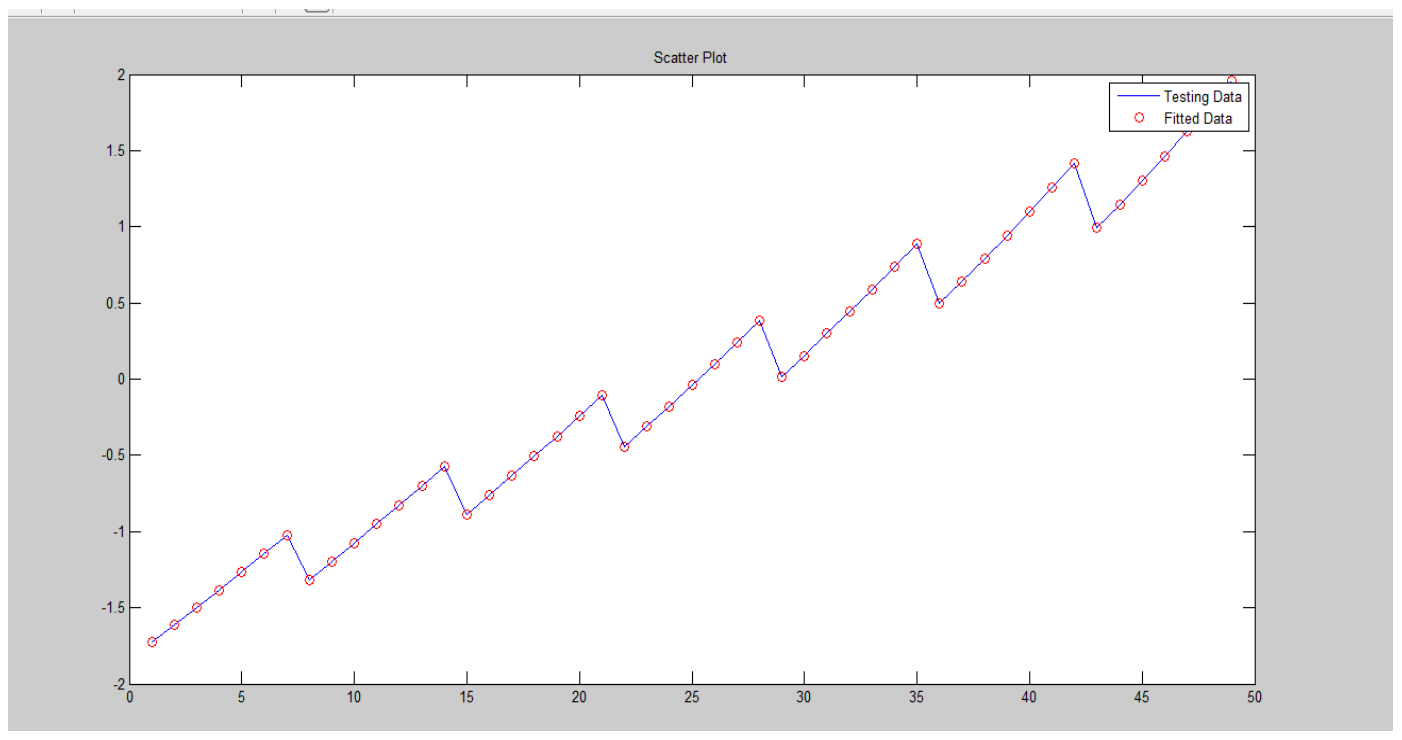

Figure 2. Graph of the Predicted y (Flow Rate of Water- Dotted Points) against Simulated Output (Solid Blue Line) over 49 Experimental Runs

\section{Discussion of Results}

The constructed OA $(49,8)$ LHD was used to develop a borehole computer experiment by scaling OA $(49,8)$ LHD and then simulate the experimental output using Equations 1 and 2, respectively. The scaled input variables and the output simulated from a computer model form the experimental results for the training datasets. The training datasets were used to fit a Gasp model. The fitted Gasp model showed that the radius $\left(r_{w}\right)$ and Hydraulic Conductivity of Borehole $\left(K_{w}\right)$ are important variables in modelling borehole experiment as given in Table 5.

The test data was also simulated using a 49-run experimental design in order to assess the accuracy of the Gasp model. These test data were used on the fitted Gasp model to predict the flow rate of water of a borehole. The predicted results interpolated the test data as shown in Figure 2. This, therefore, shows that the fitted Gasp model is very efficient.

\section{Conclusion}

A method for the statistical modelling and analysis of borehole computer experiment has been presented in this work. It is an exposition of the current trend in the design and analysis of experiments where a computer model was used to mimic a borehole 
experiment and results were obtained within the shortest possible time without having to wait for physical experimental results. This is simply called a computer experimentation approach.

The fitted Gasp model emulated the borehole computer model perfectly well and it also interpolated the test data. This work showed that the radius $\left(r_{w}\right)$ and hydraulic conductivity of borehole $\left(K_{w}\right)$ are important variables in modeling and predicting the flow rate of water of a borehole using a 49-run experimental design and Gasp model as an emulator of a borehole computer model. The estimated values for the radius $\left(r_{w}\right)$ and the hydraulic conductivity of borehole $\left(K_{w}\right)$ have the same signs, indicating that $r_{w}$ and $K_{w}$ have the same effects on the flow rate of water of a borehole. This study also showed that the $p$-values for the estimated parameters for $r(\mathrm{~m}), T_{l}\left(\mathrm{~m}^{2} / \mathrm{yr}\right), T_{u}\left(\mathrm{~m}^{2} / \mathrm{yr}\right), H_{l}(\mathrm{~m}), H_{u}(\mathrm{~m})$ and $L(\mathrm{~m})$ are very large as shown in Table 5. These six factors do not have significant linear main effects on the flow rate of water through the borehole.

The computer experimentation approach has opened up a new area in Design and Analysis of Experiments which is referred to as Design and Analysis of Computer Experiments. This area of study has permitted both greater complexity and more extensive use of mathematical models as computer models in scientific and engineering experimentations as well as in industrial processes. A Gasp model with correlation functions different from the Gaussian correlation function employed in this work could also be considered in order to compare the performance of Gasp model under different correlation functions and predict the output of the borehole computer experiment at untried inputs for future research.

\section{References}

[1] J. An and A. B. Owen, "Quasi-regression", Journal of Complexity, vol. 17, (2001), pp. 588-607.

[2] N. Dyn, D. Levin and S. Rippa, "Numerical Procedures for Surface Fitting of Scattered Data by Radial Basis Functions", SIAM Journal of Scientific and Statistical Computing, vol. 7, no. 2, (1986), 639-659.

[3] H. Fang and M. F. Horstemeyer, "Global Response Approximation with Radial Basis Functions", Journal of Engineering Optimization, vol. 38, no. 4, (2006), pp. 407-424.

[4] J. H. Friedman, "Multivariate Adaptive Regressive Splines", The Annals of Statistics, vol. 19, no. 1, (1991), pp. 1-67.

[5] W. M. Ho and Z.Q. Xu, "Applications of Uniform Design to Computer Experiments", Journal of Chinese Statistical Association, vol. 38, (2000), pp. 395-410.

[6] J. R. Koehler and A. B. Owen, "Computer experiments", Handbook of Statistics, vol. 13, (1996), pp. 261-308.

[7] M. D. Morris, T. J. Mitchell and D.Ylvisaker, "Bayesian Design and Analysis of Computer Experiments: Use of Derivatives in Surface Prediction", Technometrics, vol. 35, (1993), pp. 243-255.

[8] D.C. Montgomery, "Design and Analysis of Experiments", $5^{\text {th }}$ Edition, John Willey and Sons, Inco., New York, (2001).

[9] R. H. Myers and D. C. Montgomery, "Response Surface Methodology: Process and Product Optimization using Designed Experiments", Wiley Series in Probability and Statistics, John Wiley and Sons, New York, (1995).

[10] K.A. Osuolale, W.B. Yahya and B.L. Adeleke, "Construction of Space-filling Designs for Three Input Variables Computer Experiments", International Journal of Computer, Control, Quantum and Information Engineering, WASET, vol. 8, no. 9, (2014), pp. 1624-1628.

[11] K.A. Osuolale and W.B. Yahya, "Statistical Design and Modelling of Borehole Computer Experiment", Proceedings of the $3^{\text {rd }}$ U6 Consortium International Conference, Ilorin, Nigeria, (2015a) $6^{\text {th }}$ September $9^{\text {th }}$ September.

[12] K.A. Osuolale, W.B. Yahya and B.L. Adeleke, "Construction of Orthogonal Array-Based Latin Hypercube Design for Deterministic Computer Experiments", Annals, Computer Science Series, vol. 13, no. 1, (2015b), pp. 24-29.

[13] T. W. Simpson, T. M. Mauery, J.J. Korte and F.Mistree, "Kriging Models for Global Approximation in Simulation-Based Multidisciplinary Design Optimization”, AIAA Journal, vol. 39, (2001), pp. 22332241.

[14] S. Strogatz, “The Real Scientific Hero of 1953”, New York Times, (2003).

[15] G.G. Wang and S. Shan, "Review of Metamodeling Techniques in Support of Engineering Design Optimization" ASME Transactions, Journal of Mechanical Design, in press, (2006).

[16] B.A. Worley, "Deterministic Uncertainty Analysis, in 'ORNL - 0628" National Technical Information Service, 5285 Port Royal Road, Springfield, VA 22161, USA, (1987). 
International Journal of Advanced Science and Technology Vol.107 (2017) 